\title{
Anthropometric Study by Diagonising and Antagonizing the Feel Factor of 'Ideal Fabric' with 'Reference Fabric' for Frugal Engineering of Foundation Garments
}

\author{
K.V.P. Singh ${ }^{1}$, A. Chatterjee ${ }^{1}$, A. Das ${ }^{2}$, Raul Fangueiro ${ }^{3}$ \\ ${ }^{1}$ Department of Textile Technology NIT Jalandhar, Punjab, India; \\ ${ }^{2}$ Department of Textile Technology IIT Delhi, India; \\ ${ }^{3}$ Department of Textile Engineering University of MINHO, Portugal \\ http://dx.doi.org/10.15221/14.414
}

Keywords: Comfort, Handle, Overall desirability, 3D body scanning, DREF- III, Feel Factor.

\section{Introduction}

An attempt has been made to develop DREF III friction spun yarn after structural modifications with an objective to analyze its feasibility for apparel end use by diagnosing its quantified reduction in harshness. This harsh feel is due to the wrapper fibres of sheath which wrap the core during yarn formation (to give adequate strength). Therefore, removal of sheath is being sought by incorporating water soluble PVA fibres (Poly Vinyl Alcohol), without compromising the fundamental 'quality requirements of fabric'. [1]

An independent study is carried out to develop a regression model, for simultaneous optimization of the desirability of physiological comfort and handle related properties of a knitted fabric, made up of the modified friction spun yarn, with pre-defined constraints in desired atmospheric conditions for specific end-use and comparing it with knitted fabric made up of conventional friction spun yarn named as 'Reference fabric' [2, 3]. To optimize the desirability of value added garment, response surface methodology is used as a tool to develop a mathematical model [4].

In this work, 'Physiological Control System' (PCS) of both comfort \& handle is being upgraded to 'Engineering Control System' (ECS) by conversion of conceptual model to a mathematical model [5].Optimizing the fabric handle properties is based on the basic concept of neuroscience; artificial neuron model; physiological control system \& engineering control system. Re-optimization of both comfort and handle by reverse engineering process is done to identify the best sample with maximum overall desirability termed as an 'Ideal Fabric' to achieve value added quality garment. 'Ideal Fabric' is identified as per the synergistic target \& goal i.e. which satisfies \& saddles both the parameters of comfort and handle in a compromise zone either as per the functionality of frugal engineering or by concurrent engineering [6].

Optimization \& quantification of the responses in good zone of FAST fabric finger prints with maximum enhanced physiological comfort; to achieve a unique resultant value in a compromise zone known as an 'Overall Desirability'; as per engineering control system by developing a 'Feed Forward Back-Propagating Mathematical Model' with 'Artificial Neural Networks' (ANNs), also called cloudburst technology or parallel distributed processing systems (PDPs) and connectionist system, are intended for modeling the organizational principles of the central nervous system. Simulation and achievement of 'Ideal fabric' in terms of simultaneously re-optimized physiological comfort and handle properties in a compromise zone, for rapid prototyping of garment. [7, 8]

Indexes are used to calculate comfort for summer wear i.e. TWCI (Total wear comfort index); TPCI (Thermo-physiological comfort index); Gagge et al produced comfort sensation scale with 43 points; CALM (Comfort affect labeled magnitude) scale is also used; KVP Singh et al. quantified and optimized the overall desirability of physiological comfort and handle to achieve value added garment [9].

Anthropometric study is being done with diagnostic case study by comparing the 'Feel Factor' of 'Ideal fabric' with 'Reference fabric' and quantifying its reduction in harshness assuming body metabolism and microclimate as constant and stationary respectively as biologically inspired cloud computing capabilities of the ANN allows the cognitive and sensory task, as per database management, to be performed more easily and more satisfactorily than with conventional serial processors [10].

Antagonistic case study of 'Feel Factor' of 'Ideal fabric' with 'Reference fabric' will further be applied by using anthropometry with NX 16 3D body scanner (scheduled and controlled automated network system) on a digitized 3D model of human body to assist the spatial analysis of clothing appearance , body measurement, garment fit and for amity multitasking or rapid prototyping of apparels and medical textiles. 


\section{Methodology}

The prepared plain knitted samples of both optimized modified friction spun yarn and conventional friction spun yarn were tested and compared for analysis with an objective to quantify and optimize the desirability of both comfort and handle in order to achieve the overall desirability of an ideal fabric to achieve value added garment. [9]

To fulfill the objectives of the study, the methodology is planned out in the following manner:

1. Preparation of yarn samples.

2. Preparation of the circular knitted fabric from yarn samples.

3. Testing the knitted fabrics for desired properties.

4. Response surface method for data analysis.

5. Development of regression model equations of different responses of modified friction spun yarn.

6. Optimization of modified friction spun yarn.

7. Development of a model of an optimized modified yarn.

8. Comparative study of conventional friction yarn with optimized modified yarn \& testing of hypothesis.

9. Quantification and optimization of individual desirability of comfort and handle respectively.

10. Quantification and optimization of overall desirability of an 'Ideal fabric'.

11. Anthropometric study by diagnosing \& antagonizing the "Feel Factor' of 'Ideal with Reference fabric'.

12. Diagnostic case study to quantification of comparative reduction in harshness of friction spun yarn.

13. Antagonistic case study of 'Feel Factor' of 'Ideal fabric' with 'Reference fabric' will further be applied by using anthropometry with NX 16 3D body scanner on a digitized 3D model of human body.

\section{Desirability function}

Desirability function is used to quantify and optimize the overall desirability of comfort and handle. To optimize the desirability of value added garment from an 'Ideal Fabric, response surface methodology is used as a tool to develop a mathematical model.

It is a useful approach for optimization of multiple responses of product by using the simultaneous optimization technique.

And its value varies between $0 \leq d \leq 1$.

When response ' $Y_{i}^{\prime}$ is at its goal; then $d=1$;

And when the response is outside an acceptable region; then $d=0$

\subsection{Physiological comfort}

The state of comfort can only be achieved when the complex interactions between a range of physiological, and physical factors have taken place in a satisfactory manner i.e.

\section{Desirability of Physiological comfort $=f(a, b, c, d, e)$}

at specific atmospheric conditions with predefined constraints.

Where;

$a=$ air permeability; $b=$ thermal conductivity; $c=$ wicking; $d=$ water absorbency;

$\mathrm{e}=$ water vapour permeability are the discrete functions of physiological comfort. 
Objective evaluation of the above mentioned responses of physiological comfort as per the functional requirement of apparel and its simultaneous objective optimization is known as the desirability of physiological comfort [6].

When, there are ' $m$ ' responses then overall combined desirability is calculated by considering;

$$
\text { ' } \boldsymbol{D}_{c} \text { ' }=\left(\boldsymbol{d}_{1}, \boldsymbol{d}_{2}, \boldsymbol{d}_{3},-\cdots-\boldsymbol{d}_{5}\right)^{1 / 5} \text { (Desirability function for physiological comfort) }
$$

Where;

$\boldsymbol{D}_{\boldsymbol{c}}=$ combined desirability of overall responses of physiological comfort

$d=$ desirability of specific response ' $Y$ ' as per specific end use;

\subsection{Handle}

Desirability of Fabric handle $=f(a, b, c, d, e, f, g, h, i, j)$

At specific atmospheric conditions with predefined constraints;

Where;

$a=$ relaxation shrinkage; $b=$ hygral expansion; $c=$ formability; $d=$ extensibility; $e=$ bending rigidity; $f=$ shear rigidity; $g=$ thickness; $h=$ surface thickness; $i=$ released surface thickness; $j=$ weight; are the discrete functions of fabric handle.

When, there are ' $m$ ' responses then overall combined desirability is calculated by considering;

$$
\text { ' } D_{H} \text { ' }=\left(\boldsymbol{d}_{1}, \boldsymbol{d}_{2}, \boldsymbol{d}_{3},---\boldsymbol{d}_{10}\right)^{1 / 10} \text { (Desirability function for handle) }
$$

Where;

$D_{H}=$ combined desirability of overall responses of handle

$\mathbf{d}=$ desirability of specific response ' $Y$ ' as per specific end use;

Maximum enhancement in physiological comfort related properties of fabric with predefined constraints in desired atmospheric conditions and microclimate for specific end use along with achievement of all FAST parameters in good zone of fabric finger prints is termed as desirability of handle.

\subsection{Overall Desirability of physiological comfort and handle}

It is a useful approach for optimization of multiple responses of physiological comfort and handle by using the simultaneous optimization technique in a compromise zone. Desirability of Comfort and Handle is denoted as $D_{C} \& D_{H}$.

$$
D_{o}=\left(D_{c} \cdot D_{H}\right)^{1 / 2}
$$

Where $D_{0}=$ Overall Desirability is the desirability of value added garment and is defined as simultaneous optimization of comfort and handle as per its specific end use with known constraints It can be compared with Total Hand value by comparing individual desirability of comfort \& handle with Primary Hand values (PHV). Total Hand Value (THV) considers only handle properties but Overall Desirability $\left(D_{0}\right)$ considers both the parameters of comfort and handle [8].

\section{Simulated and Developed Products with Frugal Engineering}

\subsection{Optimized yarn}

Optimized yarn is a unique modified friction spun yarn, whose physiological comfort related responses are simultaneously optimized to avoid clamminess in summer.

\subsection{Ideal fabric}

It is a garment prepared with 'Ideal Fabric'. 'Ideal Fabric' can be defined as a fabric which satisfies all the parameters or responses of both comfort and handle simultaneously required for the specific end- use as per the desirability and concurrent engineering. 'Ideal Fabric' keeps on changing due to fabric properties required for specific end-use as per its functionality.

After the prediction of values of considered factors and responses, an optimized yarn is developed with maximum desirability value, is converted into a knitted fabric and garment. Objective evaluation 
of the above mentioned responses of physiological comfort fabric handle as per the functional requirement of the apparel and its simultaneous objective optimization to achieve a unique resultant value in a compromise zone known as the overall desirability of an 'Ideal Fabric'. Optimized value of each response of comfort and handle may be treated as 'Primary Hand Value' (PHV). The desirability of both comfort and handle may be treated as 'Total Hand Value' (THV).

Further simultaneous optimization of desirability of both comfort and handle will provide a unique resultant value in a compromise zone known as an 'Overall Desirability' co-relating both comfort and handle with concurrent engineering. 'Total Hand Value' (THV) considers only handle properties; FAST system only provides finger prints but unable to provide any unique value however 'Overall Desirability' $\left(D_{0}\right)$ considers both the parameters of comfort and handle [9].

\subsection{Reference fabric}

It is a knitted fabric made from the $70: 30$ conventional friction spun yarn [2,3].

\section{Control systems}

It can be classified as [5]

\subsection{Qualitative Control Systems}

It is collection of interconnected responses to achieve desired response. It is termed as

\section{Physiological control system (PCS)}

- PCS is an interrelated system to perform several different functions e.g. Metabolism.

- It is generally non-linear.

- It leads to qualitative conceptual model.

\subsection{Quantitative Control Systems}

It can be termed as follows;

\section{Engineering control system (ECS)}

- ECS is a specific defined task for an optimal manner e.g. Thermostat

- It can be linear or non-linear

- It is up gradation of conceptual model to mathematical model

\section{Feel Factor}

Feel factor of both the samples will be evaluated by the Fabric Feel Tester developed by IIT, Delhi and used as a tool to diagnose the variation in harshness [10].

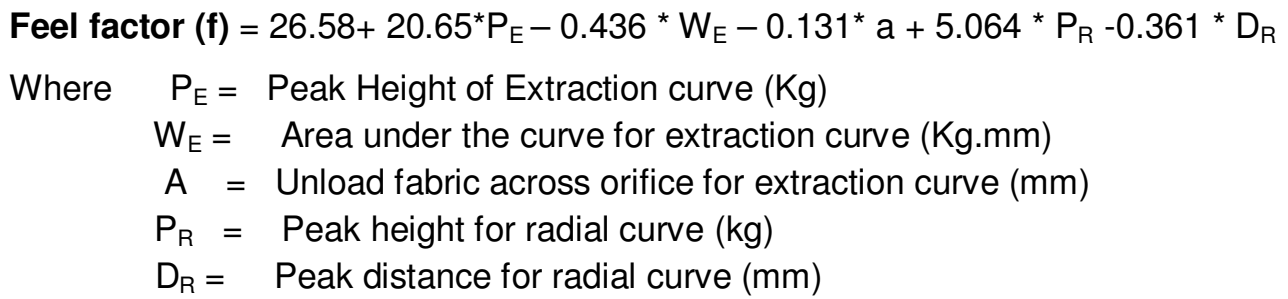

As the feel factor increases harshness increases i.e. decrease in softness and vice- versa.

\section{Anthropometric study}

This developed process along with 'Diagnostic \& Antagonistic case study' will further be applied on a digitized 3D model of human body for amity multitasking, rapid prototyping for 3D personal avatar of apparels and medical textiles Antagonistic case study of 'Feel Factor' of 'Ideal fabric' with 'Reference fabric' will be done by using anthropometry with NX 16 3D body scanner (scheduled and controlled automated network system) to assist the spatial analysis of clothing appearance, body measurement and garment fit with point cloud data and concurrent engineering $[11,12,13]$. 


\section{Result and Discussions}

\subsection{Evaluation of Overall Desirability of 'Ideal Fabric'}

As, overall desirability of 'Ideal Fabric' is;

$$
\begin{aligned}
& D_{0}=\left(D_{C} . D_{H}\right)^{1 / 2} \\
& D_{C}=0.577 \text { (desirability of comfort) } \\
& D_{H}=0.570 \text { (desirability of handle) }
\end{aligned}
$$

Therefore overall desirability

$$
\begin{aligned}
D_{0} & =(0.577 \times 0.570)^{1 / 2} \\
& =(0.32889)^{1 / 2} \\
& =0.5734
\end{aligned}
$$

If the overall desirability of fabric will be 0.5734 then we will be able to achieve the value added garment from a desired fabric known as an 'Ideal fabric'.

\subsection{Evaluation of Feel Factor}

\subsubsection{Feel factor of Reference fabric:}

(70: 30 conventional friction spun yarn i.e. Reference fabric)

Feel factor of reference fabric is evaluated as per values derived from the graph generated by 'Fabric Feel Tester' shown in Figure 1

Feel factor $(f)=26.58+20.65 * 2.777-0.436 * 0.626-0.131^{*} 0+5.064 * 3.017-0.361 * 43.094$

Feel factor $(f)=83.23$

As the feel factor increases, harshness increases.

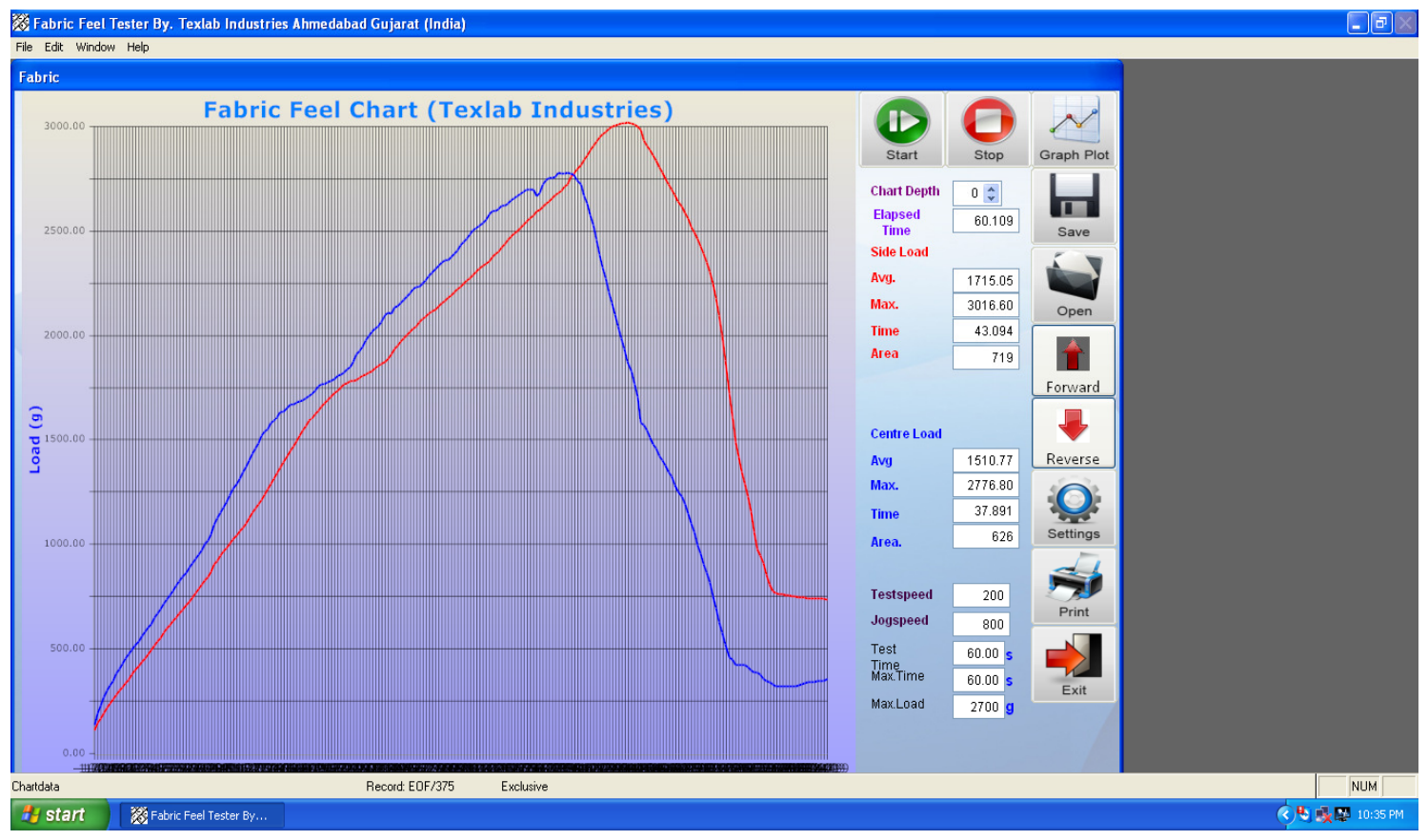

Fig. 1: Feel Factor Graph of Reference Fabric

8.2.2. Feel factor of Ideal fabric:

(Optimized friction spun yarn i.e. Ideal fabric)

Feel Factor of Ideal fabric is evaluated as per values derived from the graph generated by 'Fabric Feel Tester' shown in Figure 2

Feel Factor $(f)=26.58+20.65^{*} 1.989-0.436 * 0.443-0.131^{*} 0+5.064$ * $3.017-0.361 * 49.156$

Feel Factor $(\mathfrak{f})=64.9$

As the feel factor decreases harshness decreases i.e. increase in softness. 


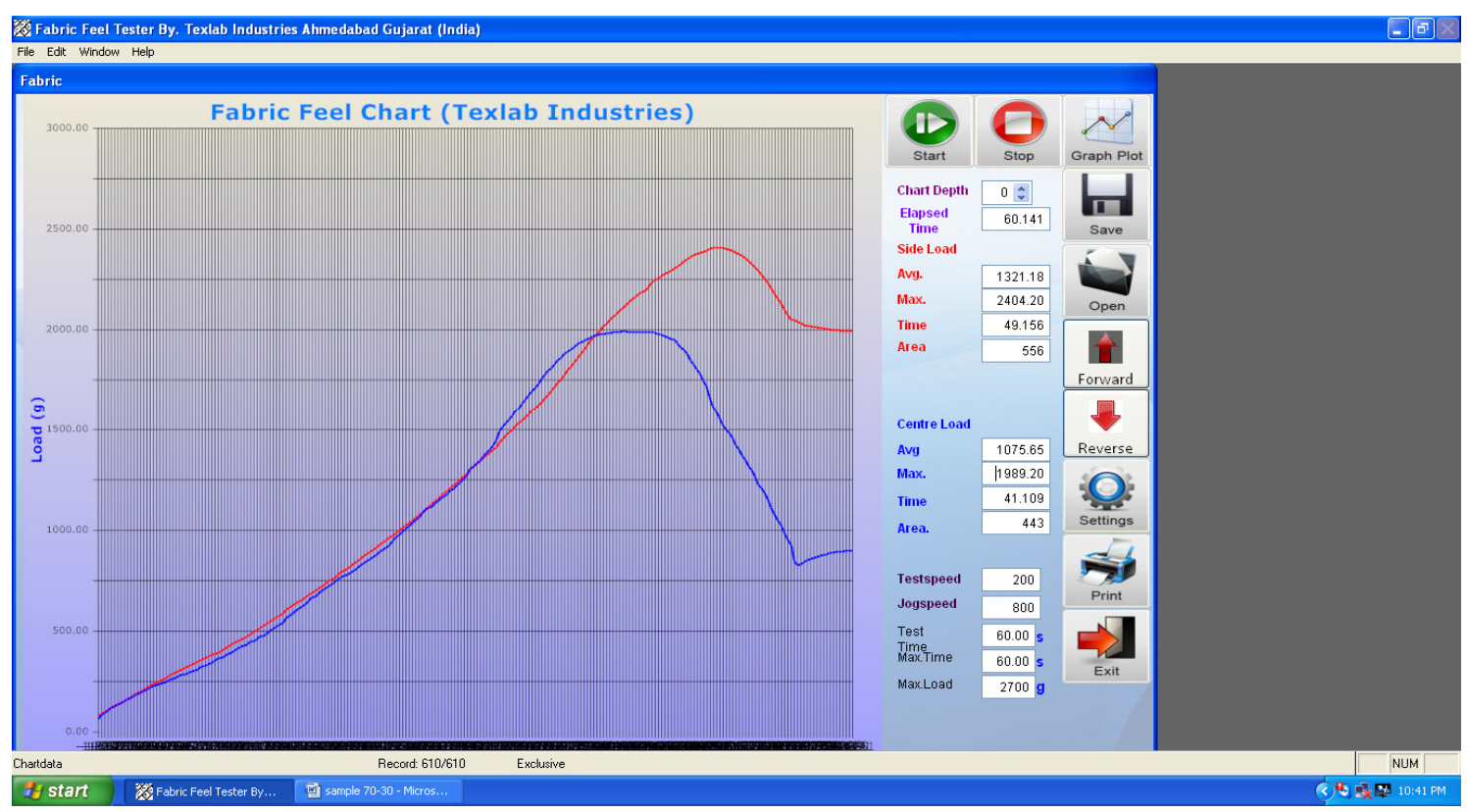

Fig. 2: Feel Factor Graph of 'Ideal Fabric'

\subsection{Anthropometric study}

\subsubsection{Antagonistic case study:}

Antagonistic case study of 'Feel Factor' of 'Ideal fabric' with 'Reference fabric' will be done by using anthropometry with NX 16 3D body scanner to assist the spatial analysis of clothing appearance , body measurement and garment fit with point cloud data and concurrent engineering; According to the degree of space allowance $(F)$ affecting somesthetic and kinesthetic sensations, garments can be classified into three types; Foundation garments $(F<0)$, perfectly fitting garments $(F=0)$ and loose garments $(F>0)$ however in this case we have assumed metabolism and microclimate as constant and stationary[13,14].

\subsubsection{Diagnostic case study:}

By comparing the feel factor of both the samples percentage reduction in harshness is being calculated

i.e. Reduction in harshness $=($ Feel Factor of 'Reference fabric') - (Feel Factor of 'Ideal fabric')

$$
((83.23-64.9) \div 83.23)^{*} 100=21.91 \%
$$

So, after structural modifications of friction spun yarn with an objective to analyze its feasibility for apparel end use, its quantified reduction in harshness is being diagnosed i.e. we are able to reduce $21.91 \%$ harshness of friction spun yarn.

\section{Conclusion}

In this work, Physiological Control System (PCS) of both comfort \& handle is being upgraded to Engineering Control System (ECS) by conversion of conceptual model to a mathematical model and achieving a unique resultant value in a compromise zone known as an 'Overall Desirability' either as per the functionality of frugal engineering or by concurrent engineering and engineering control system by developing a 'Feed Forward Back-Propagating Mathematical Model' with 'Artificial Neural Networks' (ANNs), also called Cloudburst Technology or Parallel Distributed Processing systems (PDPs) and connectionist system, are intended for modeling the organizational principles of the central nervous system.

Simulation of 'Ideal fabric' in terms of simultaneously re-optimized physiological comfort and handle properties, for its conversion into garment. In case of any technical problem during garment conversion after re-optimization; suitable solution to it should be provided. Optimization \& quantification of the responses in good zone of FAST fabric finger prints with maximum enhanced physiological comfort; to achieve an 'Ideal fabric'. 
Anthropometric study by diagnosing and antagonizing the 'Feel Factor' of 'Ideal Fabric' with 'Reference Fabric' and quantifying its reduction in harshness assuming body metabolism and microclimate as constant and stationary respectively as biologically inspired cloud computing capabilities of the ANN allows the cognitive and sensory task, as per database management, to be performed more easily and more satisfactorily than with conventional serial processors for amity multitasking or rapid prototyping.

Antagonistic case study of 'Feel Factor' of 'Ideal fabric' with 'Reference fabric' will be done by using anthropometry with NX 16 3D body scanner to assist the spatial analysis of clothing appearance , body measurement and garment fit with concurrent engineering; however in this case we have assumed metabolism and microclimate as constant and stationary

Diagnostic case study of 'Feel factor' of 'Ideal fabric' to achieve value added garment; with the garment made from 'Reference fabric' i.e. 70:30 conventional friction spun yarn; significant reduction in bending rigidity and reduction in harshness, due to removal of sheath, has taken place, keeping formability in range.

This developed process along with 'Diagnostic \& Antagonistic case study' will further be applied on a digitized 3D model of human body for amity multitasking, rapid prototyping for 3D personal avatar of apparels and medical textiles.

Scope for future work: Though there is no thumb rule or fixed philosophy;

a) However Kaizen philosophy for Software quality function deployment (SQFD) method may be used for amity multitasking or rapid prototyping of textile/garment.

b) Moreover software like Rhinoceros; SAP HANA; Stat-ease; are useful for its 3 dimensional product simulation, characterization, optimization, development with Goal oriented requirements engineering (GORE) method; Knowledge acquisition in automated specification (KAOS) method, Analytical hierarchy process and concurrent engineering.

c) Feel Factor can be used as a tool to diagnose the functionality of different fabrics as per Software quality function deployment (SQFD) method with Attributed Goal oriented requirement engineering (AGORE) method.

d) Cloudburst technology for the database management or clapper's technology of Feel factor of entire fabrics using fabric feel tester and NX 16 3D body scanner (scheduled and controlled automated network system) with point cloud data.

Acknowledgement: This work is the continuation of the continuous effort of the starred author (singhkvp@yahoo.co.uk; singhkv@nitj.ac.in) to achieve PhD and PDF under the esteemed guidance of Dr P K Banerjee, Chairman, Research Advisory Committee, Former Professor, Department of Textile Technology , IIT, Delhi,, Dr Apurba Das, Professor, Department of Textile Technology , IIT, Delhi, Dr A Chatterjee, Department of Textile Technology, Associate Professor, NIT, Jalandhar and Dr Raul Fangueiro, Professor, Department of Textile Engineering, University of MINHO, Portugal.

\section{References:}

1. Singh, K.V.P., Sinha, S. K., and Sharma, I.C., Effect of process variables on twist efficiency and some mechanical properties of Dref-III Yarn, M.Tech Thesis, Department of Textile Technology, Technological Institute of Textile and Sciences, Bhiwani, Haryana, India, 2002.

2. Singh, K.V.P., Das, A., and Chatterjee, A., Study on physiological comfort of fabrics made up of structurally modified friction spun yarns: Part I - Vapour transmission, Indian J Fibre Text Res, ,Vol.35, 2010, pp.31-37.

3. Singh, K.V.P., Das, A., and Chatterjee, A., Study on physiological comfort of fabrics made up of structurally modified friction spun yarns: Part II - Liquid transmission, Indian J Fibre Text Res, Vol.35, 2010,pp. 134-138.

4. Singh, K.V.P., Das, A., and Chatterjee, A., Modification of friction spun yarn for apparel end use, PhD Thesis, Department of Textile Technology, NIT, Jalandhar, Punjab, India, 2009

5. Michael, C. K. Khoo., Physiological control systems, Analysis, Simulation and Estimation, Prentice- Hall of India, New Delhi, ${ }^{\text {st }}$ edition, 2005, pp.1-10.

6. Douglas \& Montgomery, Design \& Analysis of Experiments, John Wiley and Sons, Singapore, $5^{\text {th }}$ edition, 2004, pp. 427-452. 
7. Wong, A. S. W., Neural Network Predictions of human physiological of clothing sensory comfort, Text Res J, Vol. 73, 2003, pp.31-37.

8. Singh, K.V.P., Das, A., and Fanguiero, R., Quantification and optimization of overall desirability to achieve value added garment, International Conference on Emerging trends in Traditional \& Technical Textiles, ,Department of Textile Technology, NIT, Jalandhar, Punjab, 11-12 ${ }^{\text {th }}$ April 2014,pp. 397-399.

9. Singh, K.V.P. , Das A and Fangueiro, R., Quantification and optimization of overall desirability to achieve value added garment, PDF Thesis, Department of Textile Engineering, University of MINHO, Campus De Azurem,Portugal,2011

10. Das, A., Objective evaluation of fabric hand characteristics using extraction principle, spring 2012Empa, May 25-27, 2012, St.Gallan, Switzerland.

11. Fan, J., W, Yu. and Hunter, L., Clothing Appearance and Fit: Science and Technology, Woodhead Publishing Limited, Cambridge, 2004.

12. Lerch, T.,3D Laser scanning technology; A model of multidisciplinary research, $\mathrm{J}$ of Textile and Apparel, Technology and Management,Vol.34(5), 2006,pp.1-22.

13. Das, A., Science in clothing comfort, Vol 1. pp 169-171, Woodhead Publishing Limited, Cambridge, 2010.

14. Traci May-Plumlee, National Textile Center Annual Report: November 2002. 